\title{
Percutaneous Endoscopic Gastrostomies - Higher Complication Rates and Preventable Admissions in Western NSW
}

\author{
Dr Shireen Kumar ${ }^{1 *}$ and Dr Geoffrey $\mathrm{Chu}^{2}$ \\ ${ }^{1} M B B S$, Orange Health Service, Orange, NSW, Australia \\ ${ }^{2}$ MBBS FRACP, Department of Medicine, Orange Health Service, NSW, Australia
}

*Corresponding author: Dr Shireen Kumar MBBS, Latrobe Regional Hospital, 10

Village Ave, Traralgon VIC 3844, Australia

Received Date: April 13, 2020

Published Date: May 12, 2020

\section{Research Letter}

Ethics: As this audit meets the statutory guidelines as detailed by the Health Records and Information Privacy act 2002 (NSW), the WNSW HREC determined that this study did not require ethics approval.

\section{Dear Editor,}

Percutaneous Endoscopic Gastrostomies (PEG) are devices that allows nutrition, medications and/or fluids to be fed directly into the stomach to minimize the risk of aspiration or obstruction in certain highrisk patients. Despite being considered a safe procedure, a number of complications can occur varying from minor (tube dislodgement, blockage or leakage, wound infection) to major (buried bumper syndrome, necrotizing fasciitis, ileus, fistula formation) [1]. The incidence of minor complications associated with PEGs have been reported as varying from $12-25 \%$ in certain studies [1,2]. However, the Agency for Clinical Innovation reports that the complication rates associated with gastrostomy formation is from $8-30 \%$, though this may vary in health care facilities for various reasons [3].

A retrospective medical record audit was conducted of patients who presented to Orange Base Hospital over a 5 year period (1st June 2012 - 31st May 2017) for PEG-related management. From existing de-identified medical records, relevant admissions were sourced using specific gastrostomy and medical codes used by the Medical Coding Unit, Interventional Radiology and Dietetics departments. Data regarding admission types, length of stay, and treating team were collected and extracted from these files. The aims of the audit were to determine the frequency and type of PEG complications, any factors contributing to these complications, and to compare current management practices of PEGs at Orange Health Service (OHS) with existing guidelines.

Over 5 years, 97 patients were identified to have presented 145 times to OHS for various PEG- related issues. $42 \%$ of these patients were female, $8 \%$ were Aboriginal/TSI and 1 was a paediatric patient. 38\% of these patients had a PEG inserted initially due to dysphagia secondary to malignancy, 19\% due to dysphagia secondary to stroke, and another $19 \%$ due to neurological or neurodegenerative disorders.

Of the 145 admissions, 51\% were for elective procedures (PEG insertion, change or removal) with nil complications present throughout the admission, while $49 \%$ of admissions were related to PEG complications. Minor complications included PEG dislodgement, damage or blockage, site leakage or infection. Major complications included entero-peritoneal fistula formation or ileus. (Figure 1) $24 \%$ of patients who presented to ED with minor complications were not admitted to the ward, and of the patients who were admitted to the ward under the General Surgical team, $13 \%$ were admitted for less than 24 hours.

A lack of continuity of care was also noted, where $96 \%$ of patients had PEGs inserted by a GI consultant or Surgical team, compared to $51 \%$ of patients who were seen by Interventional Radiology or ED Physicians on re-presentation to OHS for PEG management. 


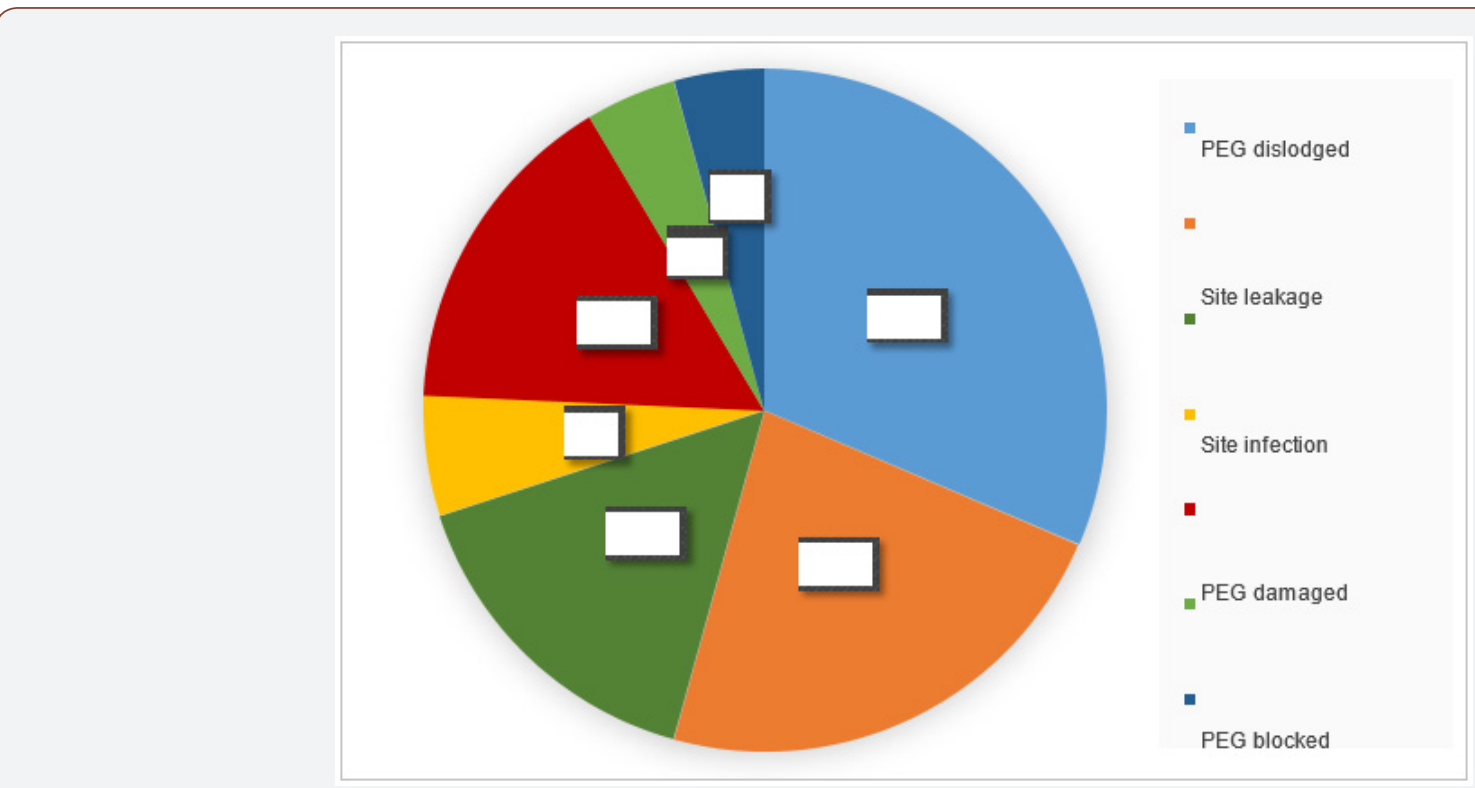

Figure 1: Breakdown of PEG Complication Admissions.

Current guidelines by the Agency for Clinical Innovation suggest an MDT approach to managing and coordinating care for patients with PEGs, which does not currently exist at OHS. The above complication rate of $49 \%$ for PEGs was also higher than the 8-30\% mentioned in ACI guidelines. PEG complication rates at OHS may be reduced by creating greater community support and a single care-coordinator. This may facilitate prompt management of PEG complications in the community and prevent future hospital admissions.

Under-resourcing is a disadvantage of regional and remote care that disproportionately affects regional populations, particularly with access to skilled and allied health personnel. The resulting lack of decentralized, MDT care models in regional health services is known to negatively affect outcomes of rural patients with cardiovascular disease and cancer [4-6].

This objective data on enteral feeding should alert regional and state health authorities that this deficiency needs to be addressed, particularly since in regional NSW there remains a lack of MDTs for patients with GI, Colorectal and Urogynaecological cancers and Inflammatory Bowel Disease, which may contribute to the higher mortality rates for patients with these conditions in regional NSW [7]. Further studies are needed to explore whether this data is mirrored in other regional areas globally that lack decentralized MDT care models in the community.

\section{Conflict of Interest}

No conflict of interest.

\section{Acknowledgement}

None.

\section{References}

1. Rahnemai Azar, A (2014) Percutaneous endoscopic gastrostomy: Indications, technique, complications and management. World Journal of Gastroenterology 20(24): 7739.

2. Vidhya C, Phoebe D, Dhina C, Jayne S, Robert F (2018) Percutaneous endoscopic gastrostomy (PEG) versus radiologically inserted gastrostomy (RIG): A comparison of outcomes at an Australian teaching hospital. Clinical Nutrition ESPEN 23: 136-140.

3. Agency for Clinical Innovation (2019) A Clinician's Guide: Caring for people with gastrostomy tubes and devices.

4. Hamilton S, Mills B, McRae S, Thompson S (2018) Evidence to service gap: cardiac rehabilitation and secondary prevention in rural and remote Western Australia. BMC Health Services Research 18(1): 64.

5. Zubrzycki J, Shipp R, Jones V (2017) Knowing, Being, and Doing: Aboriginal and Non-Aboriginal Collaboration in Cancer Services. Qualitative Health Research 27(9): 1316-1329.

6. Quigley R, Mann J, Robertson J, Bonython-Ericson S (2019) Are we there yet? Exploring the journey to quality stroke care for Aboriginal and Torres Strait Islander peoples in rural and remote Queensland. Rural and Remote Health 19(3): 4850.

7. Cancer Institute NSW (2017) Cancer control in NSW, Annual performance report 2016. Cancer Institute NSW, Sydney (NSW). 